\title{
Stochastic Resonance: A remarkable idea that changed our perception of noise
}

\author{
L. Gammaitoni ${ }^{1}$, P. Hänggi ${ }^{2}$, P. Jung ${ }^{3}$, and F. Marchesoni ${ }^{4}$ \\ 1 Department of Physics, University of Perugia, 06123 Perugia, Italy \\ 2 Institut für Physik, Universität Augsburg, Universitätsstr. 1, 86135 Augsburg, Germany \\ 3 Department of Physics and Astronomy, Ohio University, Athens, OH 45701, USA \\ 4 Dipartimento di Fisica, Università di Camerino, 62032 Camerino, Italy
}

(c) EDP Sciences, Società Italiana di Fisica, Springer-Verlag 2009

\begin{abstract}
Despite a slow start after being introduced in the 1980's, the idea of Stochastic Resonance spurred since remarkable cross disciplinary interest in natural and social sciences. Ten years after our comprehensive review [Rev. Mod. Phys. 70, 223 (1998)] Stochastic Resonance has become a research field on its own. The present Topical Issue presents the most recent applications and extensions of this surprisingly simple and still powerful idea.
\end{abstract}

PACS. 02.50.Ey Stochastic processes - 05.40.-a Brownian motion - 05.10.Gg Stochastic analysis methods

Good scientific ideas are rare, and when someone has one it sometimes takes a decade or more until the world recognizes it. The story of Stochastic Resonance [1] is a story of that type. About 30 years ago two groups in Rome [2] and Brussels [3] came up with a new idea to explain the almost periodic occurrence of the ice ages, or how a minute change in the Earth orbit around the Sun can cause a shift of the climate as dramatic as the ice ages. Their basic idea went as follows: If climate supports two stable states, one at a lower temperature (ice age) and one at a larger temperature, then fluctuations due to geodynamical events can cause random transitions between those two states. An additional, small, periodic (non random) modulation of the Earth orbit will bias the random transitions towards times where the respective transitions are most likely. If the fluctuations are too small, the transitions occur too infrequently and cannot be entrained by the modulation of the Earth orbit. If the fluctuations are too large, the random transitions would be too frequent and couldn't be entrained, either. Et voila, there is a Stochastic Resonance at an optimal level of the fluctuations!

Although it was brilliant, subsequent data did not support this idea as an explanation for the ice ages. But this was not the end of Stochastic Resonance. Quite to the contrary, the concept of Stochastic Resonance followed a dynamics of its own. It took two experimental demonstrations, respectively, on a bistable electronic circuit [4] and on a bidirectional ring laser [5], and the introduction of user friendly quantifiers [6-8] to set the new field Stochastic Resonance off to a good start.
An important turning point in this young field occurred when the resemblance of escape-time distributions in a weakly rocked bistable system [9] with interspike interval distributions of action potentials of neurons was discovered [10]. What was most striking at the time and what triggered researchers' curiosity the most was the suggestion that noise, often considered a nuisance of small influence, can become instead a very significant component of the apparatus which generates action potentials. This, combined with the notion of Stochastic Resonance, i.e. the existence of an optimal dose for the level of the fluctuations, led to a new paradigm that systems may have evolved to perform best under ambient noise levels. The field experienced a rush into exploring the role of Stochastic Resonance in noisy biological systems [11]. Hallmark research in that direction has been performed by Frank Moss and his collaborators. They have demonstrated Stochastic Resonance for the first time in a living organism. They showed that, indeed, externally added noise enhances the detection of small vibrations by the crayfish mechanoreceptor [12]. In a similar line of research Levine and Miller [13] demonstrated the benefit of noise for the cricket cercal sensory system. Remarkably, research on the beneficial role of noise for various aspects of the nervous systems, ranging from synapses [14] to cortex[15], ghost Stochastic Resonances in ensembles of neurons [16] and higher level brain function [17] and even neurorehabilitation [18] is still very active and the numbers of papers published still increasing. Typically, the biological signals that become amplified via Stochastic Resonance by ambient jittering perturbations are non-stationary in nature. 
This calls for new Stochastic Resonance quantifiers [19] with potential ramifications in information theory [20].

Representative for the role of noise and Stochastic Resonance in biologic systems are select papers of this Topical Issue [21-25]. The quest for the smoking gun proving that evolution itself has been directed by unavoidable ambient fluctuations is still being pursued, see e.g. [26].

Many of the applications of Stochastic Resonance, in particular to neuroscience, are concerned with large ensembles of coupled constituent systems. Neurons are coupled chemically through synapses, and electrically through gap junctions and form large networks. Such a network can exhibit behaviors which are drastically different from those of its constituents, depending on coupling and coupling topology. Early studies have recognized the importance of network response versus response of its parts to a weak external signal in the presence of noise [27-29], while the effects of network topology on Stochastic Resonance are the subject of ongoing research, as discussed by several contributions published in this Topical Issue [21,30-34]

From early on, the role of intrinsic noise, i.e. noise that persists because the system is subject to thermal fluctuations or, because it is small and made of few constituents, in Stochastic Resonance has been subject of investigation. After all, if nature had evolved to a state which operates optimally under ambient noise levels, the underlying sources should be intrinsic. An early pioneering study on Stochastic Resonance in periodically gated ion channels [35], the elementary building blocks of the action potential generating machinery in neurons, demonstrated Stochastic Resonance but at a noise level inconsistent with ambient levels. Bezrukov and Vodyanoy [36] reported the observation of Stochastic Resonance in a system of voltage-dependent ion channels formed by the peptide alamethicin, i.e in an synthetic ion channel. However, if contrary to what is usually expected, the thermal activation of channel proteins did not obey an Arrhenius law kinetics (as experiments indicate [37]), Stochastic Resonance would indeed occur at ambient temperatures [23].

A different line of research on the role of intrinsic noise is based on the relation between system size and noise. The smaller the number of constituent parts of a system, the larger will be the fluctuations. Hence, noise levels can be tuned to system size leading to the concept of system-size Stochastic Resonance [38-40].

Undoubtedly, the paradigm of Stochastic Resonance, originally developed to explain the ice ages has spread well beyond physics and left its fingerprints in many other scientific disciplines. The present preface is not meant as comprehensive. Indeed it is utterly incomplete as it ignores important generalizations of Stochastic Resonance to account for, among others, entropic mechanisms [41]; energetic and control questions [42-44]; quantum effects [45], even in quantum computing [46]; device development [47-49]; pattern formation [27,28], and much more.

We end this preface by closing full-circle, coming back to climate dynamics. While the big ice-ages are thought to occur every 100, 000 years, it was found that during the glacial times, sudden warmer periods occur more or less periodically at a rate of about 1500 years (the DansgaardOeschger events). The analysis of Greenland ice-core data [50] revealed a distribution of switching times between cold and warm periods, consistent with those one would expect to see if indeed the North-Atlantic climate would mimic an excitable system being driven by a weak periodic force (that likely is of solar origin), thus biasing the climate periodically to the one or other state $[51,52]$.

Furthermore, the observed transitions are extremely rapid (less than 5 years) and have refueled the discussion whether rapid climatic changes are a hallmark of human impact. Subsequently more elaborate geophysical models have been put forward to elucidate the nature of the forcing and to further substantiate the role of Stochastic Resonance [53].

\section{References}

1. L. Gammaitoni, P. Hänggi, P. Jung, F. Marchesoni, Rev. Mod. Phys. 70, 223 (1998)

2. R. Benzi, S, Sutera, A. Vulpiani, J. Phys. A 14, L453 (1981); R. Benzi, G. Parisi, A. Sutera, A. Vulpiani, Tellus 34, 10 (1982)

3. C. Nicolis, G. Nicolis, Tellus 33, 225 (1981)

4. S. Fauve, F. Heslot, Phys. Lett. A 97, 5 (1983)

5. B. McNamara, K. Wiesenfeld, d R. Roy, Phys. Rev. Lett. 60, 2626 (1988)

6. B. McNamara, K. Wiesenfeld, Phys Rev A 39, 4854 (1989)

7. P. Jung, P. Hänggi, Europhys. Lett. 8, 505 (1989); P. Jung, P. Hänggi, Phys. Rev. A 44, 8032 (1991)

8. L. Gammaitoni, F. Marchesoni, E. Menichella-Saetta, S. Santucci, Phys. Rev. Lett. 62, 349 (1989); L. Gammaitoni, F. Marchesoni, S. Santucci, Phys. Rev. Lett. 74, 1052 (1995)

9. T. Zhou, F. Moss, P. Jung, Phys. Rev. A 42, 3161 (1990)

10. A. Longtin, A. Bulsara, F. Moss, Phys. Rev. Lett. 67, 656 (1991)

11. P. Hänggi, ChemPhysChem 3, 285 (2002)

12. J. K. Douglass, L. Wilkens, E. Pantazelou, F. Moss, Nature 365, 337 (1993)

13. J.E. Levin, J.P. Miller, Nature 380, 165 (1996)

14. H. Yasuda, T. Miyaoka, J. Horiguchi, A. Yasuda, P. Hänggi, Y. Yamamoto, Phys. Rev. Lett. 100, 118103 (2008)

15. J. Mayor, W. Gerstner, Neuroreport 16, 1237 (2005)

16. A. Lopera, J.M. Buldu, M.C. Torrent, D.R. Chialvo, J. Garcia-Ojalvo, Phys. Rev. E 73, 021101 (2006)

17. P. Balenzuela, J. Garcia-Ojalvo, Chaos 15, 23903 (2005)

18. C.T. Haas, S. Turbanski, K. Kessler, D. Schmidtbleicher, NeuroRehabilitation 21, 29 (2006)

19. I. Goychuk, P. Hänggi, Eur. Phys. J. B 69, 29 (2009)

20. O.A. Rosso, C. Masoller, Eur. Phys. J. B 69, 37 (2009)

21. M. Perc, Eur. Phys. J. B 69, 147 (2009)

22. B. Spagnolo, S. Spezia, L. Curcio, N. Pizzolato, A. Fiasconaro, D. Valenti, P. Lo Bue, E. Peri, S. Colazza, Eur. Phys. J. B 69, 133 (2009)

23. Yong Woon Parc, D.S. Koh, Wokyung Sung, Eur. Phys. J. B 69, 127 (2009)

24. T. Prager, A.B. Neiman, L. Schimansky-Geier, Eur. Phys. J. B 69, 119 (2009) 
25. D. Tabarelli, A. Vilardi, C. Begliomini, F. Pavani, M. Turatto and L. Ricci, Eur. Phys. J. B 69, 155 (2009)

26. N.D. Dees, S. Bahar, F. Moss, Phys. Biol. 5, 44001 (2008)

27. P. Jung, U. Behn, E. Pantazelou, F. Moss, Phys. Rev. A 46, R1709 (1992)

28. J.F. Lindner, B. K. Meadows, W.L. Ditto, M.E. Inchiosa, A.R. Bulsara, Phys. Rev. Lett. 75, 3 (1995)

29. F. Marchesoni, L. Gammaitoni, A.R. Bulsara, Phys. Rev. Lett. 76, 2609 (1996)

30. H.S. Wio, J.A. Revelli, M.A. Rodriguez, R.R. Deza, G.G. Izus, Eur. Phys. J. B 69, 71 (2009)

31. M. Morillo, J. Gòmez-Ordòñez, J.M. Casado, J. CasadoPascual, D. Cubero, Eur. Phys. J. B 69, 59 (2009)

32. Y.-C. Lai, K. Park, L. Rajagopalan, Eur. Phys. J. B 69, 65 (2009)

33. A. Krawiecki, Eur. Phys. J. B 69, 81 (2009)

34. N. Fujiwara, J. Kurths, Eur. Phys. J. B 69, 45 (2009)

35. D. Petracchi, M. Pellegrini, M. Pellegrino, M. Barbi, F. Moss, Biophys. J. 66, 1844 (1994)

36. S.M. Bezrukov, I. Vodyanoy, Nature 378, 362 (1995)

37. H. Yanagida, R. Inoue, M. Tanaka, Y. Ito, Am. J. Physiol. Cell Physiol. 278, C40 (2000)

38. G. Schmid, I. Goychuk, P. Hänggi, Europhys. Lett. 56, 22 (2001)

39. J. W. Shuai, P. Jung, Europhys. Lett. 56, 29 (2001)

40. A. Pikovsky, A. Zaikin, M.A. de la Casa, Phys. Rev. Lett. $\mathbf{8 8}, 050601$ (2002)
41. P.S. Burada, G. Schmid, D. Reguera, J.M. Rubi, P. Hänggi, Eur. Phys. J. B 69, 11 (2009)

42. S. Lahiri, A.M. Jayannavar, Eur. Phys. J. B 69, 87 (2009)

43. J. Ma, Z. Hou, H. Xin, Eur. Phys. J. B 69, 101 (2009)

44. E. Heinsalu, M. Patriarca, F. Marchesoni, Eur. Phys. J. B 69, 19 (2009); M. Borromeo, F. Marchesoni, Eur. Phys. J. B 69, 23 (2009)

45. M. Grifoni, P. Hänggi, Phys. Rep. 304, 229 (1998)

46. A. Rivas, N.P. Oxtoby, S.F. Huelga, Eur. Phys. J. B 69, $51(2009)$

47. A.R. Bulsara, V. In, A. Kho, G. Anderson, C. Obra, P. Longhini, J. Neff, S. Baglio, B. Ando, A. Palacios, Eur. Phys. J. B 69, 109 (2009)

48. T. Dunn, D.N. Guerra, P. Mohanty, Eur. Phys. J. B 69, 5 (2009)

49. F.T. Arecchi, R. Meucci, Eur. Phys. J. B 69, 93 (2009)

50. R.B. Alley, S. Anandakrishnan, P. Jung, Paleoceanography 16, 190 (2001)

51. A. Ganopolski, S. Rahmstorf, Phys. Rev. Lett. 88, 038501 (2002)

52. S. Rahmstorf, R. Alley, Eos (Transactions, American Geophysical Union) 83, 129 (2002)

53. H. Braun, M. Christl, S. Rahmstorf, A. Ganopolski, A. Mangini, C. Kubatzki, K. Roth, B. Kromer, Nature 438, $208(2005)$ 